\title{
NOTA
}

\section{ESTADO NUTRICIONAL DA CULTURA DE CANA-DE-AÇÚCAR (CANA-PLANTA) EM EXPERIMENTOS COM ${ }^{15} \mathrm{~N}^{(1)}$}

\author{
Carlos Eduardo Faroni ${ }^{(2)}$, Paulo Cesar Ocheuze Trivelin ${ }^{(3)}$, Henrique Coutinho \\ Junqueira Franco $^{(4)}$, André Cesar Vitti ${ }^{(5)}$, Rafael Otto ${ }^{(6)}$ \& Heitor Cantarella ${ }^{(7)}$
}

\begin{abstract}
RESUMO
Estudos com isótopos estáveis são cada vez mais comuns pela qualidade e confiabilidade dos resultados obtidos e por não apresentarem risco ao homem e ao ambiente. No entanto, é necessário o uso de microparcelas para diminuir o custo do projeto. Por suas áreas serem muito reduzidas em relação à parcela onde está inserida, o objetivo deste trabalho foi comparar o estado nutricional de plantas das microparcelas com as do restante das parcelas experimentais, na fase de máximo desenvolvimento da cultura da cana-de-açúcar, em experimentos com doses de $\mathbf{N}$. Foram instalados três experimentos, no Estado de São Paulo, com três doses de N e uma testemunha, com quatro repetições, em delineamento em blocos completos casualizados. Nas parcelas, de 48 linhas de $15 \mathrm{~m}$ cada, foram instaladas microparcelas de $3 \mathrm{~m}^{2}$ com fertilizante- ${ }^{15} \mathrm{~N}$. Na época de máximo desenvolvimento da cultura foram coletadas folhas-diagnóstico para a avaliação de seu estado nutricional em todas as parcelas e microparcelas. Nessas folhas foram determinados os teores de macronutrientes, e os resultados foram submetidos à análise de variância. Foram comparados, em cada área experimental e de forma conjunta, os resultados das parcelas com os das microparcelas quanto aos teores foliares dos nutrientes e, quando significativo o efeito das doses de $\mathrm{N}$ na variação desses, foram feitas regressões. Não houve diferença entre os teores de N, P, Ca, $\mathrm{Mg}$ e S das folhas-diagnóstico entre as parcelas e as microparcelas, as quais foram
\end{abstract}

(1) Parte da Tese de Doutorado do primeiro autor apresentada ao Programa de Pós-Graduação em Solos e Nutrição de Plantas, Escola Superior de Agricultura "Luiz de Queiroz" - ESALQ/USP. Recebido para publicação em fevereiro de 2009 e aprovado em setembro de 2009.

${ }^{(2)}$ Engenheiro-Agrônomo, Doutor em Solos e Nutrição de Plantas, Pesquisador do Centro de Tecnologia Canavieira - CTC. Faz. Santo Antônio s/n, Caixa Postal 162 CEP 13400-970 Piracicaba (SP). E-mail: cfaroni@ctc.com.br

(3) Professor Associado, Laboratório de Isótopos Estáveis, Centro de Energia Nuclear na Agricultura - CENA/USP. Av. Centenário 303, Caixa Postal 96, CEP 13400-970 Piracicaba (SP). Bolsista CNPq. E-mail: pcotrive@cena.usp.br

(4) Engenheiro-Agrônomo, Pós-Doutorando do Laboratório de Isótopos Estáveis, CENA/USP. Bolsista FAPESP. E-mail: hjfranco@cena.usp.br

(5) Engenheiro-Agrônomo, Doutor em Ciências, Pesquisador Científico da APTA-Pólo Centro Sul. Rod. SP 127, km 30, Caixa Postal 28, CEP13400-970 Piracicaba (SP). E-mail: acvitti@apta.sp.gov.br

(6) Engenheiro-Agrônomo, Doutorando do Programa de Pós-Graduação em Solos e Nutrição de Plantas, Escola Superior de Agricultura "Luiz de Queiroz" - ESALQ/USP. Av. Pádua Dias 11, Caixa Postal 9, CEP 13418-900 Piracicaba-SP. Bolsista FAPESP. E-mail: rotto@esalq.usp.br

(7) Pesquisador do Instituto Agronômico de Campinas - IAC. Av. Barão de Itapura 1481, Caixa Postal 28, CEP 13020-902 Campinas (SP). E-mail: cantarella@iac.sp.gov.br 
consideradas representativas da área experimental. A adubação nitrogenada aumentou os teores dos nutrientes nas folhas-diagnóstico, o que deve ser considerado na avaliação nutricional da cultura quando este tipo de experimento for realizado.

Termos de indexação: diagnose foliar, microparcela, adubação nitrogenada, macronutrientes.

\title{
SUMMARY: NUTRITIONAL STATUS OF SUGAR CANE (PLANTED CANE) IN $15 N$ EXPERIMENTS
}

\begin{abstract}
Studies with stable isotopes are becoming more common due to the increased safety of operation and quality and reliability of results. However, the use of microplots is required to decrease the costs of such studies. Since microplots are small compared to regular plot areas, the purpose of this study was to investigate whether nutritional data based on microplot samples can adequately represent the whole area, in a comparison of the nutritional status of microplot sugar cane plants at their maximum development stage with those of the regular plots in experiments with $N$ rates. Three experiments were set up, with three $N$ rates $(40,80$, and $120 \mathrm{~kg} \mathrm{ha}^{-1} \mathrm{~N}$ ) and a control, with four repetitions, in a randomized complete block design, in the state of São Paulo. Microplots of $3 \mathrm{~m}^{2}$ containing ${ }^{15} \mathrm{~N}$-fertilizer $\left(5.04 \%\right.$ atom $\left.{ }^{15} \mathrm{~N}\right)$ were included in the main plots formed by 48 lines of sugar cane spaced $1.5 \mathrm{~m}$ apart. At the time of maximum development stage, diagnostic leaves were collected in the main and microplots to evaluate the nutritional status of plants by analyzing the total concentration of macronutrients. There were no differences in $\mathrm{N}, \mathrm{P}, \mathrm{Ca}, \mathrm{Mg}$, and $\mathrm{S}$ concentrations in the diagnostic leaves from the main and microplots, so that the latter can be considered representative of the experimental area. Higher nitrogen fertilizer rates induced increased concentrations of not only $N$, but also of $P, C a, M g$, and $S$ in the diagnostic leaves.
\end{abstract}

Index terms: diagnostic leaves, microplot, nitrogen fertilizer, macronutrients.

\section{INTRODUÇÃO}

A preocupação da sociedade mundial com o ambiente e as consequências do efeito estufa para o clima do planeta colocam em questão o uso de combustíveis fósseis, que são os grandes responsáveis pela emissão de gases poluentes na atmosfera. Vários países estão buscando reduzir o uso dessas fontes de energia pela substituição por biocombustíveis, ou uso de outras fontes menos poluentes. A cana-de-açúcar é uma das melhores opções dentre as fontes de energia renováveis atuais, com grande importância no cenário agrícola brasileiro e um futuro promissor no cenário mundial (Maule et al., 2001).

Um dos aspectos responsáveis pela alta produtividade da cana-de-açúcar é a adequada nutrição da cultura, tendo em vista a baixa fertilidade natural dos solos brasileiros (Dias, 1997). No entanto, são poucos os trabalhos desenvolvidos com cana-de-açúcar enfocando a exigência nutricional e a exportação de macronutrientes pelas variedades modernas, as quais sofreram significativa interferência genética pelo melhoramento vegetal (Prado et al., 2002).

Com enfoque no manejo nutricional da cultura, muitos trabalhos na literatura mostram a importância do N para a cana-de-açúcar. O nutriente está em apenas $1 \%$ da matéria seca total da planta, mas sua deficiência causa a redução na síntese de clorofila, de aminoácidos essenciais e da energia necessária à produção de carboidratos e esqueletos carbônicos (van Dillewijn, 1952; Alexander, 1973; Epstein, 1975). Segundo Trivelin (2000), uma das maiores limitações do meio a produtividade da cana de açúcar, nas regiões canavieiras do Brasil, é a disponibilidade de nutrientes minerais nos solos, com destaque para o $\mathrm{N}$.

Com o objetivo de entender profundamente o ciclo do N no sistema solo-planta e seu efeito no desenvolvimento e produtividade da cana-de-açúcar no Brasil, a partir de 1983, iniciaram-se os trabalhos com o uso do método do traçador isotópico para $\mathrm{N}\left({ }^{15} \mathrm{~N}\right)$ no Centro de Energia Nuclear na Agricultura, da Universidade de São Paulo. Esse método de pesquisa possibilita quantificar as taxas de transferência do nutriente nos diferentes compartimentos do sistema, podendo ser empregada em estudos quando as técnicas clássicas são insuficientes para o entendimento e a quantificação de processos no ciclo do N (Trivelin, 2000).

Antes da realização dos trabalhos nesta linha de pesquisa, pelo alto custo e baixa disponibilidade de 
isótopos na época, foi necessário definir um modelo de parcelas reduzidas que receberiam o adubo marcado com o objetivo de diminuir os custos das pesquisas. Por esse motivo, foi definido um modelo de microparcela com um segmento de linha de cana-deaçúcar com $2 \mathrm{~m}$ de comprimento que receberia o fertilizante- ${ }^{15} \mathrm{~N}$. Trivelin et al. (1994) definiram, para esse novo modelo de parcela, o comprimento da bordadura, o local de amostragem das plantas, dentro e fora da parcela, e os cálculos para determinar a recuperação do $\mathrm{N}$ derivado do fertilizante.

Apesar de hoje esse tipo de microparcela já ser de uso comum, de acordo com Souza et al. (2006), os solos cultivados com cana-de-açúcar, submetidos ao mesmo sistema de manejo, em locais com pequena variação de relevo, manifestam variabilidade espacial diferenciada de atributos como $\mathrm{pH}, \mathrm{P}, \mathrm{K}, \mathrm{Ca}, \mathrm{Mg}$, acidez potencial, capacidade de troca catiônica e saturação por bases. Essa variabilidade é condicionada pela posição dos solos na paisagem ou no declive, mesmo que o relevo mude pouco, como no caso de uma microparcela marcada com ${ }^{15} \mathrm{~N}$, em relação ao restante da parcela experimental que pode ser de até $1.000 \mathrm{~m}^{2}$ em experimentos com cana-de-açúcar. Nesse contexto, surgiu, então, o questionamento se as plantas de uma microparcela, em função da variabilidade das características químicas do solo em pequenas distâncias, poderiam apresentar absorção diferenciada, principalmente dos macronutrientes, em relação à parcela experimental na qual ela está inserida e não representá-la fidedignamente. Esse problema poderia ser mais expressivo em experimentos com doses de $\mathrm{N}$, as quais interferem no crescimento e desenvolvimento das plantas e na absorção dos demais nutrientes. Sendo assim, o objetivo deste trabalho foi comparar o estado nutricional de plantas de cana-de-açúcar de microparcelas, que receberam adubo marcado com ${ }^{15} \mathrm{~N}$, com o das parcelas experimentais, na fase de máximo desenvolvimento da cultura da cana-de-açúcar, em três experimentos com doses de N.

\section{MATERIAL E MÉTODOS}

O experimento foi realizado no Estado de São Paulo, em três áreas comerciais de cana-de-açúcar, após a reforma do canavial, com o plantio da variedade SP81 3250, nas usinas São Luiz (USL), no município de Pirassununga; Santa Adélia (USA), no município de Jaboticabal; e São Martinho (USM), no município de Pradópolis. A variedade plantada é bem aceita por suas características agronômicas, principalmente por ser produtiva e rica em açúcares e se desenvolver bem em ambientes de produção de média à baixa fertilidade do solo. Os solos das áreas experimentais são um Latossolo Vermelho-Amarelo distrófico na USL e um Latossolo Vermelho distrófico na USA, ambos de textura média, e um Latossolo Vermelho eutrófico na USM, de textura argilosa (Quadro 1).

Quadro 1. Características químicas dos solos das áreas experimentais

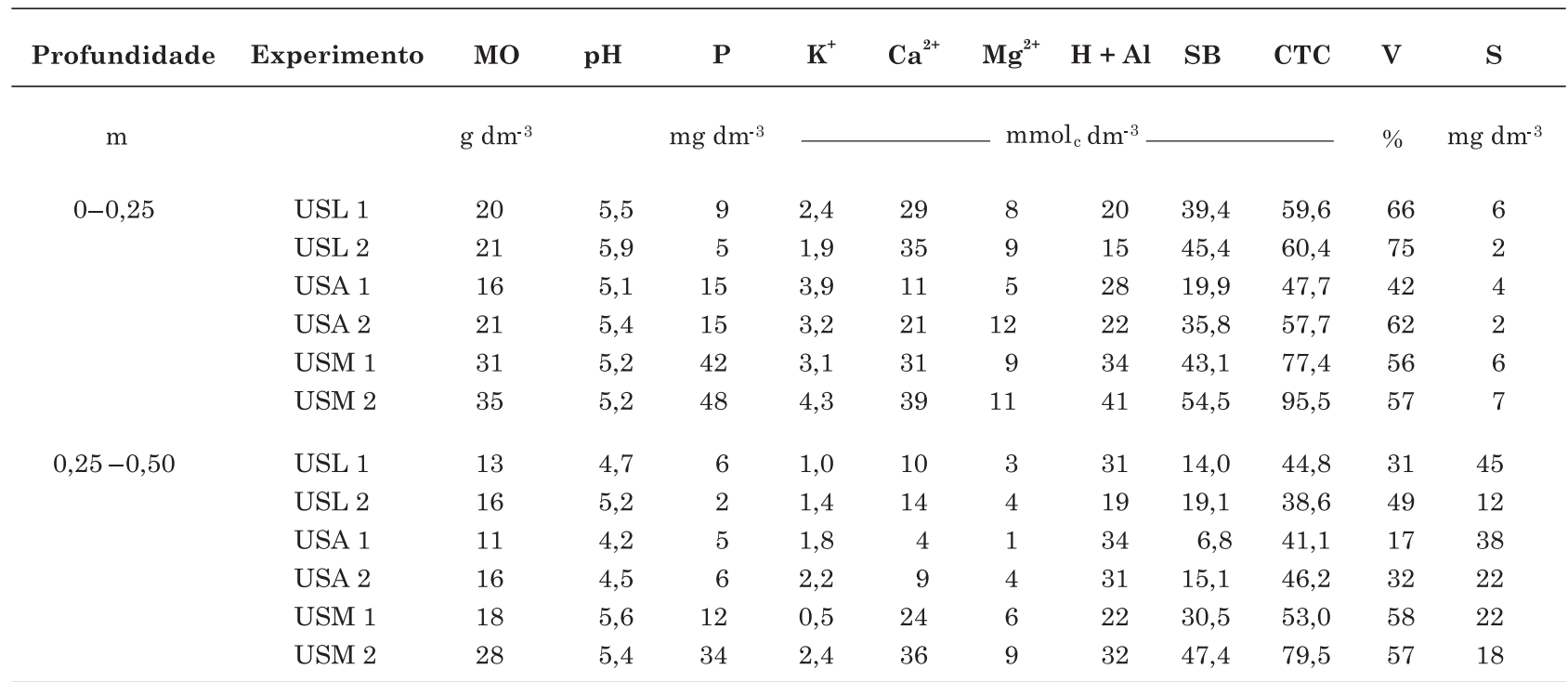

Os números 1 e 2, na frente da identificação dos experimentos, indicam a primeira (antes do plantio - fevereiro/março de 2005) e a segunda (após a colheita - setembro/outubro de 2006) época de amostragem de terra, respectivamente. $\mathrm{pH}_{-} \mathrm{CaCl}_{2}$ 0,01 mol $\mathrm{L}^{-1}$ (Raij et al., 2001). MO: matéria orgânica, método colorimétrico (Raij et al., 2001). P: extração por resina trocadora de íons e determinação por colorimetria (Raij et al., 2001). S: $\mathrm{NH}_{4} \mathrm{OAc} 0,5 \mathrm{~mol} \mathrm{~L}^{-1}$ em HOAc 0,25 mol L-1 (Vitti, 1989 ). K: extração por resina trocadora de íons e determinação por fotometria de chama (Raij et al., 2001). Ca e Mg: extração por resina trocadora de íons e determinação por espectrometria de absorção atômica (Raij et al., 2001). H + Al: determinação por potenciometria em solução tampão SMP (Raij et al., 2001). 
Na USL, as operações no preparo do solo realizadas antes do plantio foram gradeação, subsolagem, gradeação para incorporação de calcário dolomítico e gesso agrícola, ambos na dose de $2 \mathrm{Mg} \mathrm{ha}^{-1}$ cada, calculadas com base na análise de terra (Raij \& Cantarella, 1997), e gradeação leve para o nivelamento do solo antes da sulcação. A instalação do experimento ocorreu entre 21 e 24 de fevereiro de 2005 . As operações de reforma do canavial na USA foram dessecação da soqueira do ciclo anterior com aplicação de $4 \mathrm{~L} \mathrm{ha}^{-1}$ do herbicida glifosato; aração profunda e incorporação dos resíduos vegetais e de $2 \mathrm{Mg} \mathrm{ha}^{-1}$ de calcário dolomítico ao solo; e gradeação para o nivelamento do solo antes da sulcação e do plantio. O experimento foi instalado entre 4 e 8 de abril de 2005. As principais operações de preparo do solo para o plantio realizadas na USM foram dessecação da soqueira do ciclo anterior com aplicação de $5 \mathrm{~L} \mathrm{ha}^{-1}$ do herbicida glifosato, subsolagem e sulcação, não sendo necessária a aplicação de corretivos, com base na análise química de terra. A instalação do experimento ocorreu entre 1o e 4 de março de 2005.

O delineamento experimental, para cada área, foi o de blocos completos casualizados, com quatro repetições, sendo os tratamentos constituídos de três doses de N: 40, 80 e $120 \mathrm{~kg} \mathrm{ha}^{-1}$ na forma de ureia, aplicadas manualmente no fundo do sulco de plantio, mais uma testemunha sem aplicação de N. As parcelas experimentais foram compostas por 48 linhas (sulcos) de $15 \mathrm{~m}$ de comprimento cada, espaçadas entre si por $1,5 \mathrm{~m}$. No plantio, foram aplicados no fundo de sulco, além da ureia, em todas as parcelas dos experimentos, $\mathrm{KCl}$ e superfosfato simples, na dose de $120 \mathrm{~kg} \mathrm{ha}^{-1}$ de $\mathrm{K}_{2} \mathrm{O}$ e de $\mathrm{P}_{2} \mathrm{O}_{5}$.

No interior de cada parcela, foram instaladas microparcelas com dimensões de $2 \mathrm{~m}$ de comprimento e 1,5 m de largura, totalizando $3 \mathrm{~m}^{2}$ cada, sendo a ureia marcada com ${ }^{15} \mathrm{~N}\left(5,04 \%\right.$ em átomos de $\left.{ }^{15} \mathrm{~N}\right)$ aplicada nos $2 \mathrm{~m}$ de sulco. O plantio da cana-de-açúcar foi realizado com 30 mudas de canas (colmos) em $15 \mathrm{~m}$ de sulco, cruzando-se os 'pés' com as pontas das mudas, o que proporcionou uma distribuição de 17 a 20 gemas por metro linear de sulco.

$\mathrm{Na}$ fase de máximo desenvolvimento da cultura, nos dias 12, 17 e 18 de janeiro de 2006, respectivamente nos experimentos USL, USA e USM, foram coletadas, segundo Raij \& Cantarella (1997), as folhasdiagnóstico para avaliação do estado nutricional da cultura, tanto para as parcelas, quanto para as microparcelas com ${ }^{15} \mathrm{~N}$. Segundo a descrição morfológica da cultura e o sistema Kuijper de descrição das folhas, citados por van Dillewijn (1952), foram coletadas amostras compostas do terço médio de folhas $+1(\mathrm{~F}+1)$, aproximadamente $0,2 \mathrm{~m}$, excluída a nervura central. A F + 1, também denominada TVD (Top Visible Dewlap), é a primeira folha, a partir do topo da planta, que apresenta o colarinho visível.

Em cada parcela foram coletadas, aleatoriamente, quatro amostras compostas contendo cinco folhas cada.
Em cada microparcela foi coletada uma amostra composta de cinco folhas. Essas amostras foram secas em estufa de circulação forçada de ar a $65^{\circ} \mathrm{C}$ até atingirem peso constante. Após a secagem, foram moídas em moinho tipo Wiley e analisadas, segundo Malavolta et al. (1997), para determinação dos teores de N, P, K, Ca, Mg e S.

Os experimentos das USL, USA e USM foram colhidos, sem a queima dos canaviais, respectivamente, nos dias 24 de junho, 26 de julho e 10 de agosto de 2006. A estimativa da produtividade de matéria seca de cada área foi feita pela colheita das plantas de canade-açúcar da linha de $2 \mathrm{~m}$, marcada com ${ }^{15} \mathrm{~N}$ das microparcelas, e das duas linhas adjacentes a ela $\left(6 \mathrm{~m}^{2}\right)$, que representaram a microparcela, e pela colheita das plantas de quatro linhas de $15 \mathrm{~m}\left(60 \mathrm{~m}^{2}\right)$, que representaram a parcela da qual a microparcela fazia parte.

Os resultados foram submetidos à análise de variância, utilizando o teste $\mathrm{F}$ a $95 \%$ de confiança, dentro de cada área experimental, e entre elas, fazendose a análise conjunta dos experimentos (PimentelGomes \& Garcia, 2002). Para comparar os teores foliares de nutrientes das parcelas com os das microparcelas, foi realizado o teste de Tukey a $5 \%$, e para comparar o efeito de doses de $\mathrm{N}$ nos teores foliares de nutrientes usou-se a análise de regressão polinomial para os dados em que a análise de variância foi significativa.

\section{RESULTADOS E DISCUSSÃO}

Os teores de nutrientes nos solos nas três áreas experimentais (Quadro 1) variaram de médio a alto (Raij \& Cantarella, 1997), tanto antes do plantio quanto após a colheita, não representando possíveis problemas de disponibilidade de nutrientes à cultura, com exceção do P de 0 a 0,50 m e de $\mathrm{K}$ e $\mathrm{Mg}$ de 0,25 a $0,50 \mathrm{~m}$ de profundidade na USL, e do S de 0 a $0,25 \mathrm{~m}$ e de $\mathrm{P}$ e $\mathrm{Mg}$ de 0,25 a $0,50 \mathrm{~m}$ de profundidade na USA. No entanto, esses baixos teores não interferiram nos teores foliares (Quadro 2), que estavam dentro da faixa de suficiência recomendada para os canaviais do Estado de São Paulo (Raij \& Cantarella, 1997), com exceção dos teores de S em mais da metade das observações que ficaram pouco abaixo do limite mínimo de $1,0 \mathrm{~g} \mathrm{~kg}^{-1}$. Os teores de $\mathrm{K}$, na USA, superaram os da faixa considerada adequada, que são de 10 a $16 \mathrm{~g} \mathrm{~kg}^{-1}$ de K.

As produtividades das áreas experimentais, quantificadas em matéria seca da parte aérea, respectivamente para a testemunha e doses de 40, 80 e $120 \mathrm{~kg} \mathrm{ha}^{-1}$ de N, foram respectivamente de: 44.191, $44.875,48.982$, e $45.601 \mathrm{~kg} \mathrm{ha}^{-1}$ na USL, com efeito estatístico significativo para doses $(\mathrm{F}=5,146$ e $\mathrm{p}<0,05 \%) ; 48.024,48.794,49.918$, e $48.862 \mathrm{~kg} \mathrm{ha}^{-1}$ na USA, sem diferença estatística significativa entre 
os valores $(\mathrm{F}=0,322)$; e 58.237, 60.383, 59.583, e $66.711 \mathrm{~kg} \mathrm{ha}^{-1}$ na USM, com efeito estatístico significativo para doses $(F=3,32$ e $p<0,01 \%)$. Sendo assim, as doses de $\mathrm{N}$ no plantio aumentaram a produtividade em matéria seca na USL e na USM, o que não ocorreu na USA.

Não houve diferença nos teores de $\mathrm{N}$ das folhasdiagnóstico entre as microparcelas e o restante das parcelas experimentais em que estas estavam inseridas nos diferentes locais e tratamentos (Quadro 2). Nas três áreas experimentais, houve efeito significativo para as doses de $\mathrm{N}$ no teor do elemento nas folhas-diagnóstico e para a regressão dos teores. Não houve interação entre os resultados da microparcela e da parcela e as doses de N, e a média dos dois primeiros representou o comportamento da cultura nas áreas experimentais.

Na USL, observou-se uma diminuição dos teores de $\mathrm{N}$ na $\mathrm{F}+1$, com as doses de $\mathrm{N}$, mas, como houve aumento de produtividade, pode ter ocorrido um efeito de diluição, ou seja, o teor do nutriente foi diluído com o maior crescimento da planta (Jarrell \& Beverly, 1981). Por outro lado, na USA observou-se um incremento significativo do teor de $\mathrm{N}$ nas folhasdiagnóstico com as doses de $\mathrm{N}$ do plantio. Não houve efeito significativo dessas mesmas doses na produção de matéria seca da cultura, o que gerou um efeito de concentração de $\mathrm{N}$ nas plantas que pode caracterizar um consumo de luxo pela cultura ou mesmo a limitação do crescimento desta por outro fator. $\mathrm{Na}$ USM, houve aumento da produtividade e dos teores de $\mathrm{N}$ nas $\mathrm{F}+1$ com as doses de N. Esse evento pode ter explicação na maior disponibilidade de $\mathrm{N}$ nativo do solo e da matéria orgânica da área que foi superior em mais de 50 \% da encontrada na USL (Quadro 1).

Vale lembrar que a análise da folha-diagnóstico é realizada para avaliar se as plantas estão bem nutridas ou deficientes em algum elemento, não sendo possível fazer, com os teores nela determinados, uma estimativa da extração e acúmulo de nutrientes pela cultura. Dessa forma, a diluição ou concentração do nutriente na $\mathrm{F}+1$ não deve ser entendida como menor ou maior absorção do elemento, e sim se este está sendo absorvido em quantidades adequadas e balanceadas com os demais.

$\mathrm{O} \mathrm{K}$ foi o nutriente que apresentou maior variação de teor nas folhas-diagnóstico quando comparada à microparcela e à parcela na USA (Quadro 3), com efeito significativo também para a dose de N. Isso pode ser resultado de maior variação espacial da distribuição de K no solo (Souza et al., 2006), aliada à alta mobilidade do nutriente dentro da planta: por não fazer parte de nenhum componente estrutural (Malavolta, 1980; Marschner, 1995). A área da USA foi a que apresentou as maiores quantidades de $\mathrm{K}$ no solo e nas folhas, inclusive acima da faixa adequada sugerida por Raij \& Cantarella (1997) para o Estado de São Paulo. Na USL e na USM, não houve diferença nos teores de $\mathrm{K}$ nas $\mathrm{F}+1$ entre a microparcela e a parcela (Quadro 2). Na USL, houve efeito significativo das doses de $\mathrm{N}$ na concentração de $\mathrm{K}$ nas folhasdiagnóstico.

Os teores de $\mathrm{P}$ não diferiram estatisticamente entre a microparcela e a parcela nas áreas experimentais (Quadro 2). Também não houve efeito significativo das doses de $\mathrm{N}$ no teor de $\mathrm{P}$ na USL e na USA. No entanto, esse efeito foi altamente significativo na USM. Possivelmente, isso ocorreu pelo alto teor do nutriente no solo (Quadro 1), que foi absorvido em maiores quantidades estimulado pelas doses de $\mathrm{N}$, que se refletiram na produtividade e absorção de outros nutrientes. Franco et al. (2007), em experimento em vasos, e Franco (2008), em experimentos de campo, constataram que a adubação nitrogenada aumentou a extração de $\mathrm{P}$, destacando o sinergismo entre o $\mathrm{N}$ e $\mathrm{P}$ na cana-de-açúcar. De fato, o $\mathrm{P}$ é um elemento essencial para a produção de nucleotídeos e ácidos nucleicos e para a aquisição e utilização de energia como açúcares fosfatados e adenosinas fosfatadas (Epstein \& Bloom, 2006). Aliado a isso, esse nutriente é constituinte do núcleo das células, sendo essencial para a divisão celular e o crescimento e desenvolvimento do tecido meristemático do vegetal. Em vista disso, apresenta impacto decisivo na fotossíntese, síntese de proteínas e ácidos nucleicos. Portanto, a deficiência de $\mathrm{P}$ na planta, reduz a síntese de aminoácidos e proteínas (Sawan et al., 2001).

Para os teores de Ca entre a microparcela e a parcela, só houve diferença no experimento da USM, sendo maior o teor do nutriente encontrado na parcela (Quadro 2). No entanto, a faixa de variação dos teores de Ca em plantas de cana-de-açúcar é um dos mais amplos, podendo variar de 2 a $8 \mathrm{~g} \mathrm{~kg}^{-1}$ de Ca (Raij \& Cantarella, 1997). Nesse caso, a diferença das médias foi de $0,2 \mathrm{~g} \mathrm{~kg}^{-1}$ de Ca. Na USM, houve resposta linear do teor do nutriente nas folhas, e este aumentou com as doses de N. O Ca é um nutriente estrutural que se liga a polissacarídeos na parede celular, é ativador e regulador enzimático e serve como contra-íon na absorção de outros íons (Epstein \& Bloom, 2006). Por essas características, são esperados seu aumento no tecido vegetal, em pleno desenvolvimento e a alta disponibilidade de íons no solo, como é o caso da USM. $\mathrm{Na}$ USL e na USA, não houve efeito das doses de $\mathrm{N}$ no teor foliar de $\mathrm{Ca}$, sendo ele o elemento que apresentou os maiores coeficientes de variação nas áreas estudadas (Quadro 2).

Os teores de $\mathrm{Mg}$ e S não diferiram entre a parcela e a microparcela em nenhuma das áreas. No experimento da USA, não houve efeito da dose de $\mathrm{N}$ no teor de $\mathrm{Mg}$ foliar, ocorrendo o mesmo na área da USL com o S (Quadro 2). Na USL e na USM, houve aumento linear e altamente significativo no teor de Mg na F + 1 com as doses de N. Esse fato pode ser explicado pela participação do $\mathrm{Mg}$ na clorofila e na sua atividade de ativador enzimático (Epstein \& Bloom, 2006). Como esses experimentos apresentaram respostas às doses de $\mathrm{N}$ com aumento de produtividade 
Quadro 2. Teores de N, K, P, Ca, Mg e S em folhas +1 nas três áreas experimentais, na microparcela e na parcela

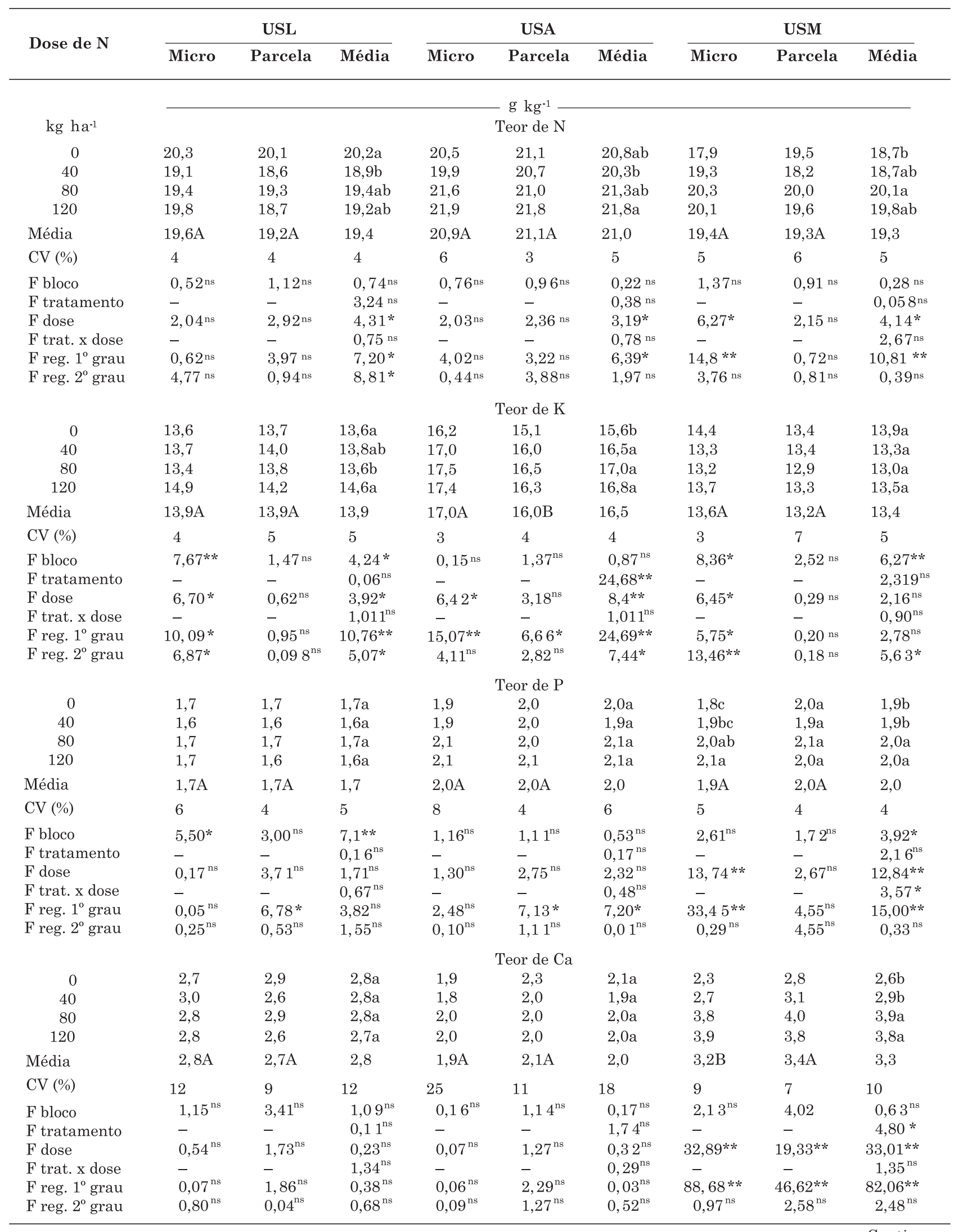


Quadro 2. Continuação

\begin{tabular}{|c|c|c|c|c|c|c|c|c|c|}
\hline \multirow{2}{*}{ Dose de N } & \multicolumn{3}{|c|}{ USL } & \multicolumn{3}{|c|}{ USA } & \multicolumn{3}{|c|}{ USM } \\
\hline & Micro & Parcela & Média & Micro & Parcela & Média & Micro & Parcela & Média \\
\hline & \multicolumn{9}{|c|}{$\mathrm{g} \mathrm{kg}^{-1}$} \\
\hline $\mathrm{kg} \mathrm{ha}^{-1}$ & & & & & Teor de Mg & & & & \\
\hline 0 & 1,3 & 1,3 & $1,3 \mathrm{~b}$ & 1,2 & 1,4 & $1,3 \mathrm{a}$ & 1,0 & 1,2 & $1,1 \mathrm{~b}$ \\
\hline 40 & 1,3 & 1,2 & $1,2 \mathrm{~b}$ & 1,2 & 1,3 & $1,2 \mathrm{a}$ & 1,3 & 1,3 & $1,3 \mathrm{~b}$ \\
\hline 80 & 1,3 & 1,3 & $1,3 \mathrm{~b}$ & 1,3 & 1,3 & $1,3 \mathrm{a}$ & 1,8 & 1,8 & $1,8 \mathrm{a}$ \\
\hline 120 & 1,5 & 1,5 & $1,5 \mathrm{a}$ & 1,2 & 1,3 & $1,3 \mathrm{a}$ & 1,8 & 1,8 & $1,8 \mathrm{a}$ \\
\hline Média & $1,3 \mathrm{~A}$ & $1,3 \mathrm{~A}$ & 1,3 & $1,2 \mathrm{~A}$ & $1,3 \mathrm{~A}$ & 1,3 & $1,5 \mathrm{~A}$ & $1,5 \mathrm{~A}$ & 1,5 \\
\hline CV (\%) & 5 & 8 & 7 & 15 & 11 & 13 & 9 & 17 & 10 \\
\hline F bloco & $4,4^{*}$ & $2,05^{\mathrm{ns}}$ & $3,99^{*}$ & $0,33^{\mathrm{ns}}$ & $1,22^{\mathrm{ns}}$ & $0,39^{\mathrm{ns}}$ & $4,52 *$ & $0,57^{\mathrm{ns}}$ & $4,91^{* *}$ \\
\hline F tratamento & - & - & $0,01^{\mathrm{ns}}$ & - & $\begin{array}{l}1,24 \\
-\end{array}$ & $3,14^{\text {ns }}$ & - & - & $1,45^{\mathrm{ns}}$ \\
\hline $\mathrm{F}$ dose & $8,67^{* * *}$ & $6,35^{*}$ & $12,08^{* *}$ & $0,33^{\text {ns }}$ & $0,78^{\text {ns }}$ & $0,30^{\mathrm{ns}}$ & $35,49^{* *}$ & $5,11^{*}$ & $50,27^{* *}$ \\
\hline $\mathrm{F}$ trat. $\mathrm{x}$ dose & - & - & $1,35^{\mathrm{ns}}$ & - & - & $0,71^{\mathrm{ns}}$ & - & - & $0,68^{\mathrm{ns}}$ \\
\hline F reg. $1^{\circ}$ grau & $17,97^{* *}$ & $11,92^{* *}$ & $39,20 * *$ & $0,35^{\mathrm{ns}}$ & $1,62^{\mathrm{ns}}$ & $0,01^{\mathrm{ns}}$ & $92,02^{* *}$ & $13,80^{* * *}$ & $89,26 * *$ \\
\hline \multirow[t]{2}{*}{ F reg. $2^{\circ}$ grau } & $6,04^{*}$ & $5,03^{*}$ & $25,00^{* *}$ & $0,28^{\mathrm{ns}}$ & $0,25^{\mathrm{ns}}$ & $0,04^{\mathrm{ns}}$ & $6,31^{*}$ & $0,85^{\mathrm{ns}}$ & $2,36^{\mathrm{ns}}$ \\
\hline & \multicolumn{9}{|c|}{ Teor de $\mathrm{S}$} \\
\hline 0 & 1,5 & 1,4 & $1,5 \mathrm{a}$ & 1,4 & 1,4 & $1,4 \mathrm{~b}$ & 1,2 & 1,4 & $1,3 b$ \\
\hline 40 & 1,3 & 1,3 & $1,3 \mathrm{a}$ & 1,5 & 1,5 & $1,5 \mathrm{~b}$ & 1,3 & 1,4 & $1,3 \mathrm{~b}$ \\
\hline 80 & 1,5 & 1,4 & $1,5 \mathrm{a}$ & 1,7 & 1,6 & $1,6 \mathrm{a}$ & 1,3 & $\begin{array}{l}1,4 \\
1,3\end{array}$ & $1,3 \mathrm{~b}$ \\
\hline 120 & 1,5 & 1,4 & $1,5 \mathrm{a}$ & 1,8 & 1,8 & $1,8 \mathrm{a}$ & 1,5 & 1,5 & $1,5 \mathrm{a}$ \\
\hline Média & $1,5 \mathrm{~A}$ & $1,4 \mathrm{~A}$ & 1,4 & $1,6 \mathrm{~A}$ & $1,6 \mathrm{~A}$ & 1,6 & $1,3 \mathrm{~A}$ & $1,4 \mathrm{~A}$ & 1,4 \\
\hline CV (\%) & 11,7 & 8,7 & 10,3 & 6,9 & 6,1 & 6,2 & 6,4 & 6,8 & 6,6 \\
\hline F bloco & $2,15^{\mathrm{ns}}$ & $1,81^{\mathrm{ns}}$ & $3,12^{\mathrm{ns}}$ & $0,41^{\mathrm{ns}}$ & $0,26^{\mathrm{ns}}$ & $0,47^{\mathrm{ns}}$ & $1,69^{\mathrm{ns}}$ & $0,63^{\mathrm{ns}}$ & $1,36^{\mathrm{ns}}$ \\
\hline F tratamento & - & - & $0,51^{\mathrm{ns}}$ & - & - & $1,62^{\text {ns }}$ & - & - & $3,92^{\text {ns }}$ \\
\hline $\mathrm{F}$ dose & $1,52^{\text {ns }}$ & $0,59^{\mathrm{ns}}$ & $2,05^{\mathrm{ns}}$ & $14,04^{* *}$ & $8,63^{* *}$ & $23,89^{* *}$ & $6,03^{*}$ & $2,67^{\mathrm{ns}}$ & $7,00^{* *}$ \\
\hline F trat. $x$ dose & - & - & $0,361^{\mathrm{ns}}$ & - & - & $2,67^{\mathrm{ns}}$ & - & - & $1,36^{\mathrm{ns}}$ \\
\hline F reg. $1^{\circ}$ grau & $0,32^{\mathrm{ns}}$ & $0,07^{\mathrm{ns}}$ & $0,06^{\text {ns }}$ & $39,31^{* *}$ & $23,45^{* *}$ & $41,43^{* *}$ & $16,47^{* *}$ & $1,69^{\mathrm{ns}}$ & 9,956 * \\
\hline F reg. $2^{\circ}$ grau & $1,058^{\mathrm{ns}}$ & $1,05^{\mathrm{ns}}$ & $0,83^{\mathrm{ns}}$ & $0,20^{\text {ns }}$ & $1,74^{\mathrm{ns}}$ & $0,08^{\text {ns }}$ & $0,78^{\mathrm{ns}}$ & $5,65^{*}$ & $1,99^{\mathrm{ns}}$ \\
\hline
\end{tabular}

Médias seguidas da mesma letra minúscula nas colunas e maiúsculas nas linhas dentro de cada área experimental não diferem entre si pelo teste de Tukey a $5 \%$. ** e *; equivalem a significâncias de 99 e $95 \%$ respectivamente. ${ }^{\text {ns: }}$ não significativo.

de matéria seca, é de se esperar que o Mg seja mais absorvido para fazer parte da maior quantidade de clorofila, ou por fazer parte dos mecanismos fisiológicos da planta.

$\mathrm{Na}$ USA e na USM, houve aumento linear e altamente significativo no teor de $\mathrm{S}$ foliar com as doses de N. O S nas plantas encontra-se, em sua maior parte, nas formas de cisteína, metionina, proteínas, coenzimas, tiamina, biotina e coenzima A, e sua deficiência pode interferir na síntese de proteínas regulatórias, essenciais para a manutenção da atividade celular (Malavolta, 1980; Marschner, 1995). Com isso, a deficiência de $\mathrm{S}$ pode limitar a resposta da planta à adubação nitrogenada, o que pode ter ocorrido nas áreas experimentais, principalmente na USA, já que as plantas de sua área experimental foram as que apresentaram os maiores teores de $\mathrm{N}$ nas folhas, e os teores de $\mathrm{S}$, se não estão abaixo da faixa considerada adequada, estão próximos do limite inferior de 1,5 $\mathrm{g} \mathrm{kg}^{-1}$ de S (Raij \& Cantarella, 1997).

$\mathrm{Na}$ análise conjunta dos experimentos, o único nutriente que diferiu entre a microparcela e a parcela foi o K, possivelmente pelas razões já citadas para o experimento USA, que tiveram um impacto maior quando os dados foram analisados conjuntamente (Quadro 3). As doses de $\mathrm{N}$ do plantio tiveram efeito significativo no acúmulo de todos os nutrientes nas folhas-diagnóstico da cana-de-açúcar. Com a análise conjunta foi possível identificar o comportamento geral dos nutrientes no solo e sua absorção pelas plantas na região estudada. No entanto, a análise isolada dos experimentos é importante, pois identifica quando os resultados são muito expressivos e podem exercer maior impacto (peso) na análise.

Outro fato que pode ter influenciado nessas análises, não somente na conjunta, mas também em cada experimento, é a folha-diagnóstico a ser amostrada. Prado et al. (2002) relataram alguns problemas entre a diagnose foliar de cana-de-açúcar e seu real estado nutricional, possivelmente por terem usado a terceira folha com o colarinho visível $(\mathrm{F}+3)$, como recomendado por muitos autores (Gallo, 1968; Raij, 1991; Malavolta et al., 1997). A folha foi coletada precocemente, aos quatro meses de idade da planta, podendo haver 
Quadro 3. Teores de macronutrientes nas folhas +1 na análise conjunta dos experimentos, na microparcela marcada, na parcela e na média

\begin{tabular}{|c|c|c|c|c|c|c|c|c|c|}
\hline Dose de N & Micro & Parcela & Média & Micro $P$ & Parcela & Média & Micro & Parcela & Média \\
\hline \multicolumn{10}{|l|}{$\mathrm{kg} \mathrm{ha-1}$} \\
\hline & \multicolumn{3}{|c|}{ Teor foliar de $\mathrm{N}$} & \multicolumn{3}{|c|}{ Teor foliar de K } & \multicolumn{3}{|c|}{ Teor foliar de $\mathrm{P}$} \\
\hline 0 & 19,5 & 20,2 & $19,9 \mathrm{a}$ & 14,7 & 14,1 & $14,4 \mathrm{a}$ & 1,8 & 1,9 & $1,8 \mathrm{ab}$ \\
\hline 40 & 19,4 & 19,2 & $19,3 \mathrm{~b}$ & 14,7 & 14,5 & $14,6 \mathrm{a}$ & 1,8 & 1,8 & $1,7 \mathrm{~b}$ \\
\hline 80 & 20,4 & 20,1 & $20,3 \mathrm{a}$ & 14,7 & 14,4 & $14,5 \mathrm{a}$ & 1,9 & 1,9 & $1,9 \mathrm{a}$ \\
\hline 120 & 20,6 & 20,0 & $20,3 \mathrm{a}$ & 15,3 & 14,6 & $14,9 \mathrm{a}$ & 1,9 & 1,9 & $1,9 \mathrm{a}$ \\
\hline Média & $20,0 \mathrm{~A}$ & $19,9 \mathrm{~A}$ & 19,9 & $14,8 \mathrm{~A}$ & $14,4 \mathrm{~B}$ & 14,6 & $1,9 \mathrm{~A}$ & $1,9 \mathrm{~A}$ & 1,9 \\
\hline CV (\%) & 6 & 6 & 5 & 12 & 10 & 5 & 11 & 10 & 6 \\
\hline F local & - & - & $87,03^{* *}$ & - & - & $35,88^{* *}$ & - & - & $35,79^{* *}$ \\
\hline $\mathrm{F}$ tratamento & - & - & $0,34^{\mathrm{ns}}$ & - & - & $8,48^{* *}$ & - & - & $1,15^{\mathrm{ns}}$ \\
\hline $\mathrm{F}$ dose & $2,55^{\mathrm{ns}}$ & $1,63^{\mathrm{ns}}$ & $6,12^{* *}$ & $0,362^{\mathrm{ns}}$ & $0,28 \mathrm{~ns}$ & $2,37^{\mathrm{ns}}$ & $1,60^{\mathrm{ns}}$ & $0,48^{\text {ns }}$ & $5,36 * *$ \\
\hline F reg. $1^{\circ}$ grau & $6,09 *$ & $0,04 \mathrm{~ns}$ & $3,60 *$ & $0,62 \mathrm{~ns}$ & $0,63^{\mathrm{ns}}$ & $1,26^{\mathrm{ns}}$ & $4,25^{*}$ & $0,30 \mathrm{~ns}$ & $3,71^{*}$ \\
\hline \multirow[t]{2}{*}{ F reg. $2^{\circ}$ grau } & $0,99 \mathrm{~ns}$ & $1,72^{\mathrm{ns}}$ & $1,33^{\mathrm{ns}}$ & $0,42^{\mathrm{ns}}$ & $0,05^{\mathrm{ns}}$ & $0,13 \mathrm{~ns}$ & $0,18^{\mathrm{ns}}$ & $0,21 \mathrm{~ns}$ & $0,17^{\mathrm{ns}}$ \\
\hline & \multicolumn{3}{|c|}{ Teor foliar de $\mathrm{Ca}$} & \multicolumn{3}{|c|}{ Teor foliar de $\mathrm{Mg}$} & \multicolumn{3}{|c|}{ Teor foliar de $\mathrm{S}$} \\
\hline 0 & 2,3 & 2,7 & $2,5 \mathrm{~b}$ & 1,1 & 1,3 & $1,2 \mathrm{~b}$ & 1,4 & 1,4 & $1,4 \mathrm{bc}$ \\
\hline 40 & 2,5 & 2,6 & $2,5 \mathrm{~b}$ & 1,3 & 1,2 & $1,2 \mathrm{~b}$ & 1,4 & 1,4 & $1,4 \mathrm{c}$ \\
\hline 80 & 2,8 & 2,9 & $2,9 a$ & 1,5 & 1,5 & $1,5 \mathrm{a}$ & 1,5 & 1,4 & $1,5 \mathrm{~b}$ \\
\hline 120 & 2,9 & 2,8 & $2,8 \mathrm{a}$ & 1,5 & 1,5 & $1,5 \mathrm{a}$ & 1,6 & 1,6 & $1,6 \mathrm{a}$ \\
\hline Média & $2,6 \mathrm{~A}$ & $2,7 \mathrm{~A}$ & 2,7 & $1,3 \mathrm{~A}$ & $1,4 \mathrm{~A}$ & 1,4 & $1,5 \mathrm{~A}$ & $1,5 \mathrm{~A}$ & 1,5 \\
\hline CV (\%) & 27 & 25 & 12 & 17 & 16 & 11 & 13 & 9 & 8 \\
\hline F local & - & - & $263,6^{* *}$ & - & - & $10,21^{*}$ & - & - & $13,03^{* *}$ \\
\hline $\mathrm{F}$ tratamento & - & - & $3,66^{\mathrm{ns}}$ & - & - & $3,27^{\mathrm{ns}}$ & - & - & $0,06 \mathrm{~ns}$ \\
\hline $\mathrm{F}$ dose & 1,88 ns & $0,66^{\mathrm{ns}}$ & $9,75 * *$ & $6,43 * *$ & $4,87 * *$ & $23,60 * *$ & $4,80 * *$ & $3,82^{*}$ & $13,57 * *$ \\
\hline F reg. $1^{\circ}$ grau & $5,21^{*}$ & $0,76^{\mathrm{ns}}$ & $5,20^{*}$ & $17,88^{* *}$ & $10,82^{* *}$ & $28,55^{* *}$ & $12,45^{* *}$ & $7,15^{*}$ & $19,66^{* *}$ \\
\hline F reg. $2^{\circ}$ grau & 0,13 ns & $0,01^{\mathrm{ns}}$ & $0,11^{\mathrm{ns}}$ & $0,48^{\mathrm{ns}}$ & $0,33 \mathrm{~ns}$ & $0,02 \mathrm{~ns}$ & $0,38^{\mathrm{ns}}$ & $4,27^{*}$ & $2,91^{\mathrm{ns}}$ \\
\hline
\end{tabular}

Médias seguidas da mesma letra minúscula nas colunas e maiúsculas nas linhas dentro de cada área experimental não diferem entre si pelo teste de Tukey a $5 \%$. ${ }^{* *}$ e *; equivalem a significâncias de 99 e $95 \%$ respectivamente. ${ }^{\text {ns: }}$ não significativo.

divergência quanto à parte da planta a ser amostrada e à interpretação do seu estado nutricional. A opção, neste trabalho, por amostrar a F + 1 levou em conta a época de amostragem das folhas entre nove e 10 meses de idade da planta, período de máximo desenvolvimento e extração de nutrientes do solo. A $\mathrm{F}+1$ é a folha madura mais nova e com maiores chances de não ter sofrido injúrias mecânicas ou causadas por pragas e doenças, as quais podem alterar seus teores normais de nutrientes.

\section{CONCLUSÕES}

1. Não houve diferença no estado nutricional das plantas de cana-de-açúcar entre as parcelas e as microparcelas marcadas com ${ }^{15} \mathrm{~N}$ no ciclo de canaplanta, podendo ser utilizadas para avaliação nutricional em experimentos com o isótopo de $\mathrm{N}$.

2. Os teores de N, P, Ca, Mg e S nas folhasdiagnóstico $(\mathrm{F}+1)$ colhidas na fase de máximo desenvolvimento da cultura de cana-de-açúcar não diferiram entre locais amostrados: microparcelas e parcelas experimentais.
3. A adubação nitrogenada aumentou os teores dos nutrientes $\mathrm{N}, \mathrm{P}, \mathrm{Ca}, \mathrm{Mg}$ e $\mathrm{S}$ nas folhas-diagnóstico $(\mathrm{F}+1)$ da cana-de-açúcar (cana planta) na época de máximo desenvolvimento da cultura.

\section{AGRADECIMENTOS}

À FAPESP, pelo financiamento da pesquisa, e às Usinas São Luiz, Santa Adélia e São Martinho, pela cessão das áreas experimentais e apoio no desenvolvimento do projeto.

\section{LITERATURA CITADA}

ALEXANDER, A.G. Sugar cane physiology: A comprehensive study of Saccharum souce-to-link system. Amsterdam, Elsevier, 1973. 752p.

DIAS, F.L.F. Relação entre a produtividade, clima, solos e variedades de cana-de-açúcar, na Região Noroeste do Estado de São Paulo. Piracicaba, Escola Superior de Agricultura "Luiz de Queiroz", 1997. 64p. (Tese de Mestrado) 
EPSTEIN, E. Nutrição mineral das plantas: Princípios e perspectivas. São Paulo, EDUSP, 1975. 341p.

EPSTEIN, E. \& BLOOM, A. Nutrição mineral de plantas: Princípios e perspectivas. 2.ed. Londrina, Planta, 2006. 403p.

FRANCO, H.C.J. Eficiência agronômica da adubação nitrogenada de cana-planta. Piracicaba, Escola Superior de Agricultura Luiz de Queiroz, 2008. 127p. (Tese de Doutorado)

FRANCO, H.C.J.; BOLOGNA, I.R.; FARONI, C.E.; VITTI, A.C. \& TRIVELIN, P.C.O. Acúmulo de macronutrientes em cana-de-açúcar em função da adubação nitrogenada e dos resíduos culturais incorporados ao solo no plantio. Bragantia, 66:521-526, 2007.

GALLO, J.R.; HIROCE, R. \& ALVAREZ, R. Levantamento do estado nutricional de canaviais de São Paulo pela análise foliar. Bragantia, 27:365-382, 1968.

JARRELL, W.M. \& BEVERLY, R.B. The dilution effect in plant nutrition studies. Adv. Agron., 34:197-224, 1981.

MALAVOLTA, E. Elementos de nutrição mineral de plantas. São Paulo, Agronômica Ceres, 1980. 251p.

MALAVOLTA, E.; VITTI, G.C. \& OLIVEIRA, S.A. Avaliação do estado nutricional das plantas: Princípios e aplicações. Piracicaba, Associação Brasileira para Pesquisa da Potassa e do Fosfato, 1997. 319p.

MARSCHNER, H. Mineral nutrition of higher plants. 2.ed. San Diego, Academic Press, 1995. 889p.

MAULE, R.F., MAZZA, J.A. \& MARTA JR., G.B. Produtividade agrícola de cultivares de cana-de-açúcar em diferentes solos e épocas de colheita. Sci. Agric., 58:295-301, 2001.

PIMENTEL-GOMES, F. \& GARCIA, C.A. Estatística aplicada a experimentos agronômicos e florestais: Exposição com exemplos e orientações para uso de aplicativos. Piracicaba, FEALQ, 2002. 309p.
PRADO, R.M.; FERNANDES, F.M. \& NATALE, W. Calcário e escória de siderurgia avaliados por análise foliar, acúmulo, e exportação de macronutrientes em cana-de-açúcar. Sci. Agric., 59:129-135, 2002.

RAIJ, B.van. Fertilidade do solo e adubação. Piracicaba, Agronômica Ceres, 1991. 343p.

RAIJ, B.van; ANDRADE, J.C.; CANTARELLA, H. \& QUAGGIO, J.A., eds. Análise química para avaliação da fertilidade de solos tropicais. Campinas, Instituto Agronômico de Campinas, 2001. 285p.

RAIJ, B.van \& CANTARELLA, H. Outras culturas industriais. In: RAIJ, B.van; CANTARELLA, H.; QUAGGIO, J.A. \& FURLANI, A.M.C., eds. Recomendações de adubação e calagem para o Estado de São Paulo. Campinas, Fundação IAC, 1997. p.233-243. (Boletim, 100)

SAWAN, Z.M.; HAFEZ, S.A. \& BASYONY, A.E. Effect of phosphorus fertilization and foliar application of chelate zinc and calcium on seed, protein and oil yields and oil properties of cotton. J. Agric. Sci., 136:191-198, 2001.

SOUZA, Z.M.; MARQUES JR, J.; PEREIRA, G.T. \& BARBIERI, D.M. Small relief shape variations influence spatial variability of soil chemical attributes. Sci. Agric., 63:161168, 2006

TRIVELIN, P.C.O. Utilização do nitrogênio pela cana-deaçúcar: Três casos estudados com o uso do traçador ${ }^{15} \mathrm{~N}$. Piracicaba, Universidade de São Paulo, 2000. 143p. (Tese de Livre-Docência)

TRIVELIN, P.C.O.; LARA CABEZAS, W.A.R.; VICTORIA, R.L. \& REICHARDT, K. Evaluation of a ${ }^{15} \mathrm{~N}$ plot design for estimating plant recovery of fertilizer nitrogen applied to sugar cane. Sci. Agric., 51:226-234, 1994.

van DILLEWIJN, C. Botany of sugarcane. Waltham, Chronica Botanica, 1952. 371p.

VITTI, G.C. Avaliação e interpretação do enxofre no solo e na planta. Jaboticabal, FUNEP, 1989. 37p. 